\title{
The Interconnections of the LHC Cryomagnets at CERN: Strategy Applied and First Results of the Industrialization Process
}

\author{
J. Ph. Tock, F. Bertinelli, P. Fessia, A. Jacquemod, A. Musso, and A. Poncet
}

\begin{abstract}
The final interconnections of the LHC superconducting magnets in the underground tunnel are performed by a contractor on a result-oriented basis. A consortium of firms was awarded the contract after competitive tendering based on a technical and commercial specification.

The implementation of the specific technologies and tooling developed and qualified by CERN has required an important effort to transfer the know-how and implement the follow-up of the contractor.

This paper summarizes the start-up phase and the difficulties encountered. The organization and management tools put in place during the ramping-up phase are presented. In addition to contractual adaptations of the workforce, several configuration changes to the workflows were necessary to reach production rates compatible with the overall schedule and with the different constraints: availability of magnets, co-activities with magnets transport and alignment, handling of non-conformities, etc. Also the QA procedures underwent many changes to reach the high level of quality mandatory to ensure the LHC performance.

The specificities of this worksite are underlined and first figures of merit of the learning process are presented.
\end{abstract}

Index Terms-Industrialization process, interconnections, LHC, superconducting magnets.

\section{INTRODUCTION}

$\mathbf{T}$ HE Large Hadron Collider (LHC) is a 27-km long circular particle accelerator, presently under final assembly at CERN, Geneva. The interconnection of the main components is taking place in an underground tunnel, of $3.8 \mathrm{~m}$ diameter. (Fig. 1).

The final assembly phase of the superconducting components, mainly cryomagnet-to-cryomagnet and cryomagnet-tocryogenic line, requires the inter-connection of the vacuum, electrical, cryogenics and thermal insulation systems [1]. In this paper, the term interconnection (IC) includes all connecting activities of the lines between two cryomagnets. The main ones are:

— connection of beam lines (working pressure below $10^{-13}$ mbar) by TIG welding,

— inductive soldering of the main busbars ( 6 off $13 \mathrm{kA}$ splices per IC) [2],

Manuscript received August 28, 2007.

The authors are with the Accelerator Technology Department of CERN, The European Laboratory for Particle Physics, 1211 Geneva 23, Switzerland (e-mail: Jean-Philippe.Tock@cern.ch).

Color versions of one or more of the figures in this paper are available online at http://ieeexplore.ieee.org.

Digital Object Identifier 10.1109/TASC.2008.920621

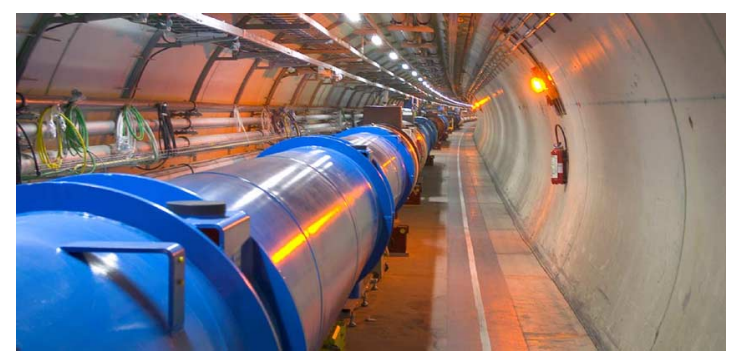

Fig. 1. View of the LHC tunnel filled with cryomagnets.

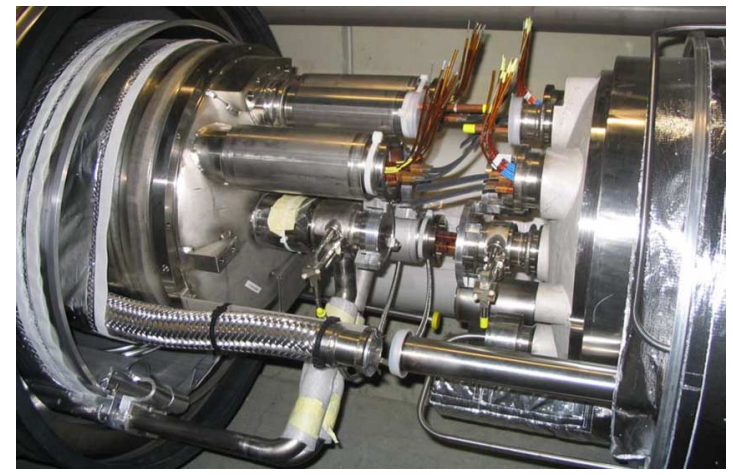

Fig. 2. Cryomagnets ready for IC.

- ultrasonic welding of the auxiliary and spool pieces busbars (up to 66 off 600 A splices) [3], [4],

- connection of the cryogenic lines (about 25 leak tight stainless steel TIG welds),

- installation of multi-layer thermal insulation (3 blankets amounting to 40 layers) and thermal screens (aluminum and stainless steel),

- closure of the insulation vacuum.

Fig. 2 shows two adjacent magnets ready for interconnection and after definitive closure in Fig. 3.

\section{SCOPE OF WORK}

The LHC is made of eight arcs and eight insertions (Fig. 4) [5]. Table I summarizes the quantity of interconnections between cryogenic components and also with the cryogenic line (the so-called jumpers) per zone and gives also the corresponding quantity of different types, which is inversely proportional to the standardization.

The arc is composed of 23 identical FODO cells, each comprising 6 cryodipoles and 2 Short Straight Sections (SSS) housing quadrupoles. 1464 interconnections of 5 types between cryomagnets and 176 jumpers are required to assemble the 8 arcs. Although complex, the interconnection work can 


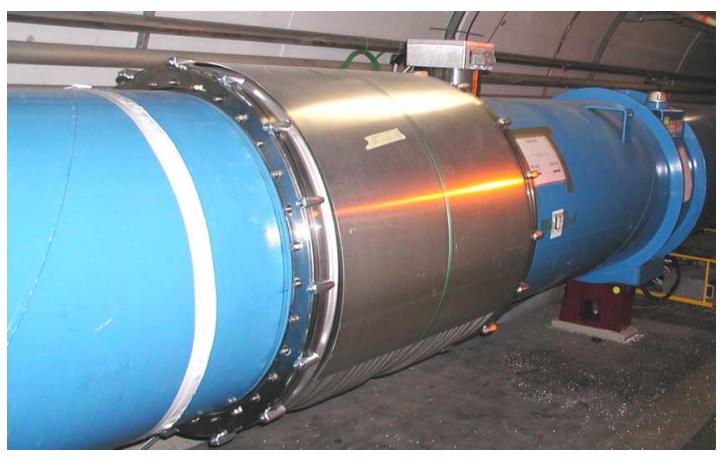

Fig. 3. Closed IC between cryodipoles.

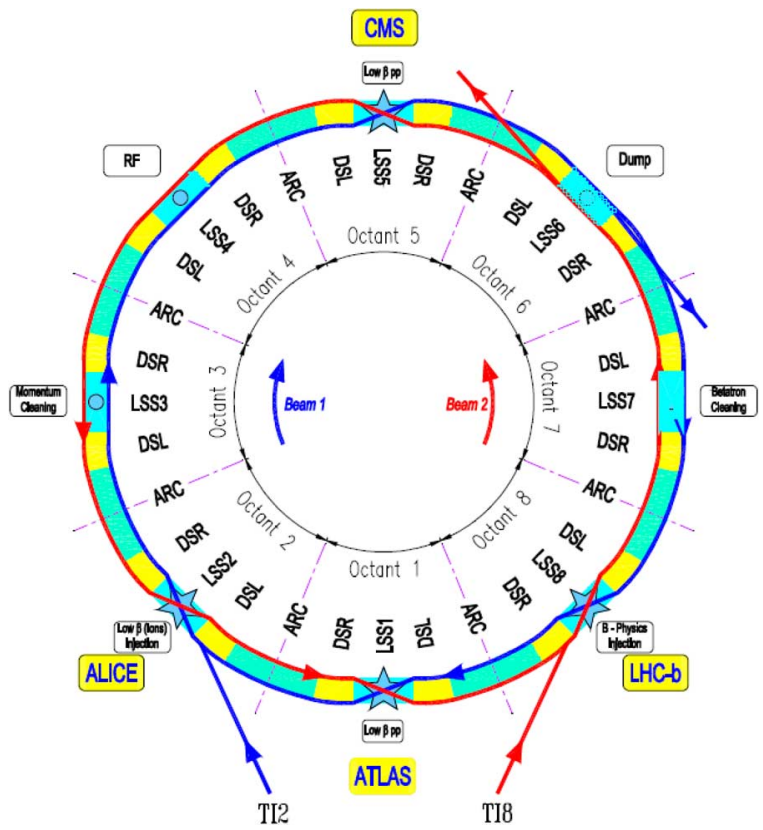

Fig. 4. Schematic layout of the LHC.

TABLE I

TYPES OF INTERCONNECTIONS

\begin{tabular}{ccccc}
\hline \hline Zone & Arc & DS & LSS & TOTAL \\
\hline $\begin{array}{c}\text { Cryomagnets IC } \\
\text { (types) }\end{array}$ & $\begin{array}{c}1464 \\
(5)\end{array}$ & $\begin{array}{c}182 \\
(15)\end{array}$ & $\begin{array}{c}26 \\
(16)\end{array}$ & $\begin{array}{c}1672 \\
(34)\end{array}$ \\
\hline $\begin{array}{c}\text { Other components IC } \\
\text { (types) }\end{array}$ & 0 & 33 & 108 & 141 \\
\hline $\begin{array}{c}\text { Jumpers } \\
\text { (types) }\end{array}$ & 176 & 32 & 102 & 310 \\
\hline \hline
\end{tabular}

be considered as a repetitive activity that can be effectively specified and divided into work packages to be carried out, on a result-oriented basis, by an external company.

The insertion region (Fig. 5) is a part of the ring between two arcs, consisting of one central Long Straight Section (LSS), flanked by a Dispersion Suppressor (DS) on each side [6].

In the DS zones, 215 interconnections of 30 types and 32 jumpers are carried out. As most of the activities are similar to those of the arc, except for the connection of the line- $\mathrm{N}$ auxiliary bus bar line [3], a mixed approach has been chosen: the standard activities are carried out by the contractor on a result-oriented basis while the non standard ones are carried out in collaboration between CERN staff and a dedicated industrial team on a resource-oriented basis.

In the LSS [7], 134 interconnections of 86 types and 102 jumpers are performed. Even if this work is highly non standard, it involves similar technologies, applied in the same difficult environment as in the arcs and DS regions. Therefore it was considered more efficient to have the work executed by the same contractor and to profit in this way from the experience acquired in the arcs, but on a resource-oriented basis, under the direct supervision and responsibility of the CERN team that has designed, developed and validated all the interconnection procedures.

\section{CONTRACTING PROCESS}

Applying this philosophy, and conforming to the management decision to have this work carried out by an external contractor, a market survey aiming at identifying qualified potential bidders was launched by CERN in Spring 2002 among 70 firms. In April 2003, a technical and commercial specification was sent out to one firm and four consortia, qualified during the market survey process. It was written on a result-oriented basis for the standard parts, representing the major volume of work, and resource-oriented with provision for variations and adaptation to the actual needs for the non-standard ones. This allows coping with non-conformities and unforeseen additional work. A 2-days bidders conference was organized to explain thoroughly the specification and to provide detailed answers to the questions from the potential bidders. The offers were analyzed to check their conformity with the specification in the technical, management and commercial domains. Subsequently, a consortium of three firms was awarded the contract in September 2003.

\section{TOOLING}

The IC work implies the application of validated technologies and specifically developed tooling, meeting the tight and unique constraints of the LHC tunnel and achieving the high level of performance required by the LHC machine. For each specific tooling, a functional specification was established and integrated in the call for tender. During the bidders conference, the CERN-developed tooling was also presented to the contractor as an example to demonstrate the feasibility of the specification. The goal was to prompt the contractor to improve the tooling and make it more industrial, more adapted to series production and more efficient to use. There was room for improvement with respect to the CERN tooling because these were laboratory tools, with a lot of built-in flexibility and designed to find the optimum operating parameters and to assemble "only" $110 \mathrm{~m}$ of the LHC machine during the full-scale validation on a test string called STRING 2 [8].

Presenting an existing set of tooling fulfilling the functional specification was also important to avoid overestimation of its cost by the contractor.

The first phase of the contract was devoted to the procurement of the tooling in collaboration with CERN. During this phase, the contractor has heavily benefited from the considerable experience acquired during the development phase, thus avoiding traps already identified, thanks to a strong involvement of the 


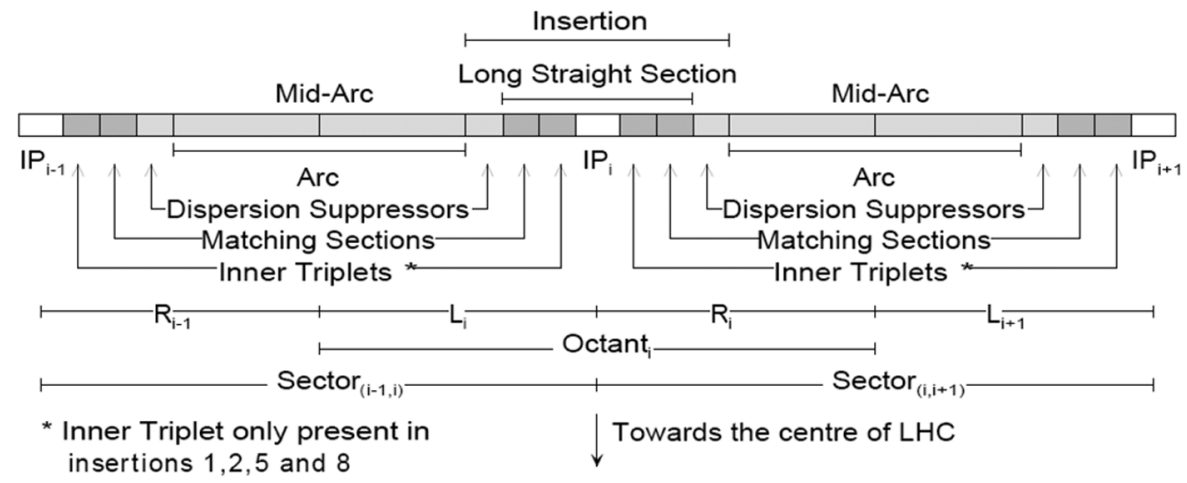

Fig. 5. Definition of the collider parts.

CERN team. This approach, though necessitating important resources, was successful, as only minor adaptations had to be made to the contractor procured tooling during the production phase.

The technical requirements could only be reached thanks to a comprehensive technology transfer at the beginning of the production, a deep commitment of all parties involved and a very close follow-up from CERN supervision throughout the whole production.

\section{Evolution OF THE CONTRACT}

Following serious difficulties encountered with the installation of the cryogenic distribution line, a recovery plan was defined in order to minimize their impact [9]. As can be seen on the tunnel cross section (Figs. 1 and 6), the cryogenic line must be installed and validated before installation of the cryomagnets. Major interventions on the cryoline with the cryomagnets in place are difficult and time consuming because of the poor access. The cryoline difficulties induced therefore delays to the installation of the first cryomagnets and consequently to the start of their interconnection in the tunnel. To accelerate the repair of the cryogenic distribution line, it was decided to bring into play the resources available through the interconnection contractor, by invoking the contractual provision for additional works. These extra resources eased the installation of the cryogenic line and, as a side effect, provided some experience to the contractor for work in the difficult tunnel environment and also in managing the interfaces and the various intervening teams.

The first interconnections became available in sector 8-1 in May 2005, more than one year later than initially planned. In order to minimize the impact of this delay, the number of work fronts was increased from 4 to 7 , requiring additional sets of tooling and extra supervision staff. Also, the initial schedule was foreseeing a sequence of activities where no co-activity would occur between transport, cryogenic distribution line installation and interconnection. The increased parallelism [9] applied in view of minimizing the impact of the additional delays has generated unforeseen co-activity resulting in working conditions far from those specified. Furthermore, as it was not possible to work in chains of magnets, non-optimum interconnection sequences had to be followed, with additional intermediate tests. The first

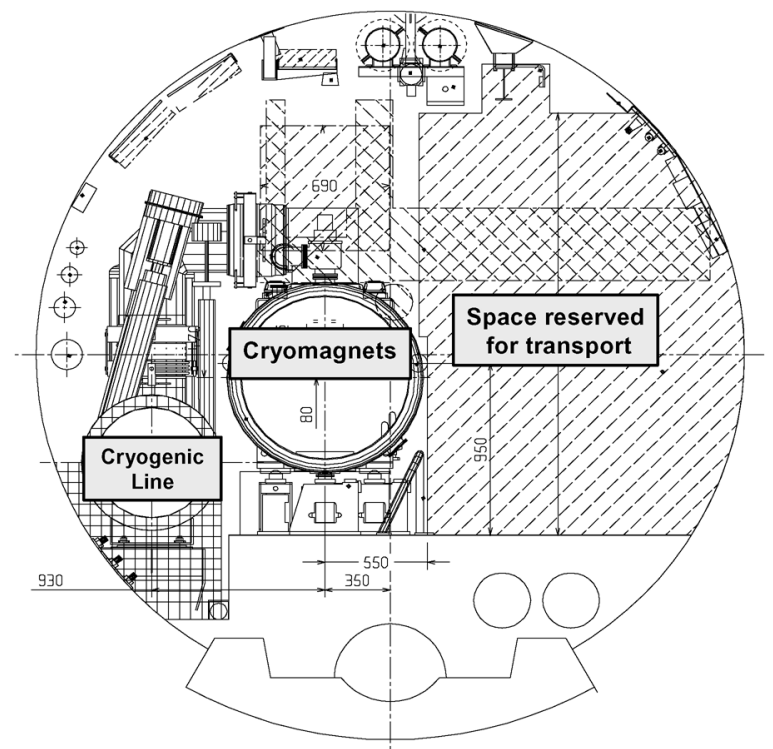

Fig. 6. LHC tunnel cross section.

sector, $8-1$, was heavily affected by co-activity and consequently efficiency was low. As soon as all components were in place and available for interconnection, the top priority was placed on interconnecting the second sector (7-8) in order to pressure test it and then immediately afterwards to cool it down in order to validate its cryogenics performance.

Starting from the third sector, 4-5, the situation improved considerably where:

- the intricate workflow of activities was optimized,

- the relations between the various intervening teams were fully understood by all the actors,

- the co-activity was reduced to the minimum,

- chains of magnets were available, making series production work possible.

All this, together with the learning process, allowed the initially foreseen interconnection production rate to be reached and even exceeded. More than $90 \%$ of the interconnection work is today completed. A preliminary analysis of the learning process by sector is shown in Fig. 7. There, the total interconnection time to complete one sector (1/8 of the LHC machine) is given for the 8 sectors in arbitrary units, normalized at 1 for the last sector. 


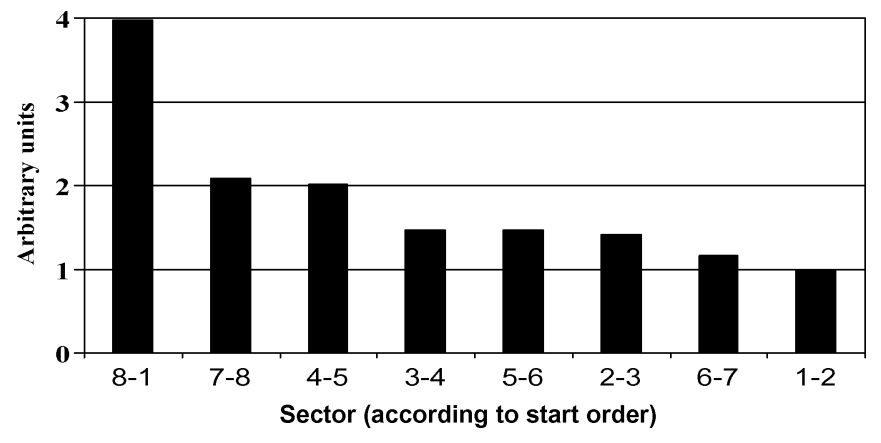

Fig. 7. Start-to-finish time for each sector.

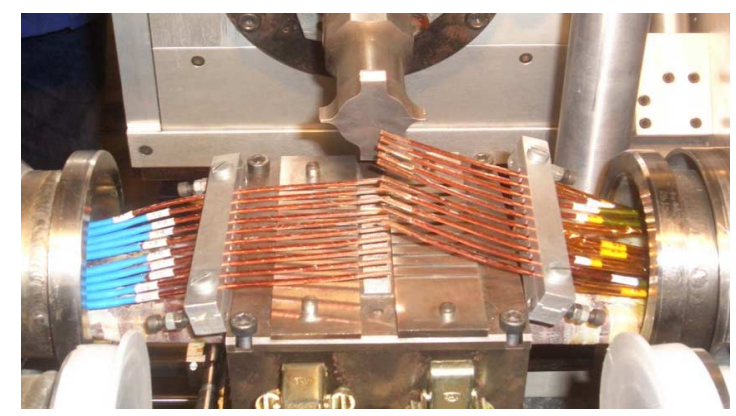

Fig. 8. Spool pieces cabling.

This analysis is preliminary; for the 5 last sectors, the scheduled end date is taken into account.

From this, it can be seen that the production rate has been increased by more than 4 with respect to the first sectors; that the priority put on the sector 7-8 had a positive effect on this sector. From the 4 th sector (3-4) onwards, the gain was low $(33 \%)$. The last sector (1-2) is benefiting from all the available resources so becoming the one most rapidly completed. This evolution incorporates not only the learning process but also the availability of resources and the effects of the priorities defined by management.

\section{QUALITY INDICATORS}

Many parameters linked to quality have been monitored constantly during production. The overall analysis is not yet completed. An example is given below.

During interconnection, cabling takes place in 2 locations, for the spool pieces (Fig. 8) (20 off splices) and in the line-N box (Fig. 9) (up to 48 off splices) [3]. There are 406 such line-N boards having almost each a different scheme. The cables are labeled; it is checked independently several times. Despite the care shown by the cabling operators, some errors occurred. However an efficient electrical quality control allowed errors to be identified and corrected well before the circuits were powered. The quantity of non-conformities (NC) is proportional to the inattentiveness of the operators for this specific activity. The production rate was increased but it had to be ensured that this was not detrimental to quality. Fig. 10 shows the evolution through the sectors of the two relevant families of NCs.

In Fig. 10, for the line $\mathrm{N}$ cabling, it can be seen that the number of NC increased dramatically in sector 3-4. This was

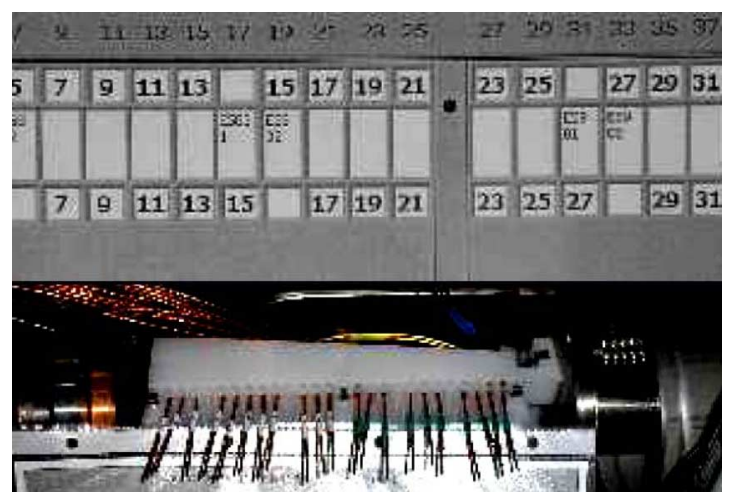

Fig. 9. Line $\mathrm{N}$ cabling board and the corresponding scheme.

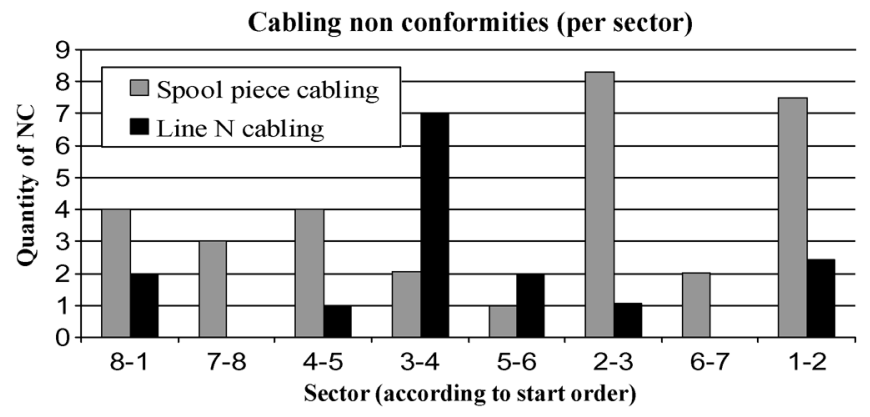

Fig. 10. Evolution of cabling non-conformities.

linked to the introduction of a new team and has triggered a reinforcement of the supervision of the operators to come back within acceptable limits. This was effective in the next sectors.

For the spool piece cabling NC, no obvious link with the increase of the production rate can be evidenced.

Other quality indicators, like the percentage of leak tight welds for example, are presently under analysis. It can not be concluded that the quality decreased with the increase of production rate.

\section{CONCLUSION}

Supported by a beforehand exhaustive validation of the technologies, tooling and procedures internally at CERN [8] and backed up by a team of dedicated experts, the signature of a procurement contract on a fixed cost basis after competitive tendering was a key to incite the contractor to optimize its resources and to work with CERN in the overall benefit of the project. The flexible strategy tailored to the peculiarities of each zone proved to be efficient.

This very preliminary analysis will be expanded and detailed in a further paper when all the data will be available and a thorough study will have been performed. Comparisons with a similar contract in charge of the assembly of the LHC cryomagnets at CERN will be drawn [10].

Lessons learnt and results obtained in the frame of this contract will be applicable to future similar projects and could be used for the on-going studies.

\section{ACKNOWLEDGMENT}

The authors thank warmly their many colleagues at CERN for their effort and for keeping the required high level of quality up 
to the end of the interconnection work, foreseen for September 2007.

The authors thank particularly the IEG (Ineo, Endel, Gti) industrial consortium in charge of the assembly of the interconnections for their flexibility and for the collaborative atmosphere they promoted and that has prevailed throughout.

\section{REFERENCES}

[1] J. P. Tock, A. Jacquemod, A. Poncet, and B. Skoczen, "The interconnections of the LHC cryomagnets," in Proc. Particle Accelerator Conf., Chicago, 2001, pp. 616-618.

[2] J. P. Tock, A. Jacquemod, A. Poncet, F. Schauf, and B. Skoczen, "Inductive soldering of the junctions of the main superconducting busbars of the LHC," in Proc. 6th European Conf. Applied Superconductivity, Sorrento, Sep. 2003, pp. 447-454.

[3] D. Bozzini, F. Laurent, S. Russenschuck, B. Skoczen, and J. P. Tock, "Electro-mechanical aspects of the interconnection of the LHC superconducting corrector magnets," in Proc. European Particle Accelerator Conf., Lucerne, 2004, pp. 1645-1647.
[4] A. Jacquemod, F. Laurent, J. P. Tock, J. M. Balaguer, and L. Vaudaux, "Qualification and start of production of the ultrasonic welding machines for the interconnection of the LHC superconducting bus bars," in Proc. 2005 IEEE Conf. Magnet Technology 19, Genoa, Jun. 2006, vol. 16, no. 2, pp. 1729-1732.

[5] O. Bruning et al., The LHC Main Ring, LHC Design Rep.; CERN-2004-03, vol. 1 [Online]. Available: http://www.ab-div. web.cern.ch/ab-div/Publications/LHC-DesignReport.html

[6] R. Saban, LHC Quality Assurance Plan, LHC-PM-QA-204 rev 1.1 [Online]. Available: http://www.lhc-proj-qawg.web.cern.ch/lhc-projqawg/LHCQAP/\#200

[7] C. Garion, I. Slits, and J. P. Tock, "Installation and quality assurance of the interconnections between cryo-assemblies of the LHC long straight sections," in Proc. European Particle Accelerator Conf., Edinburgh, 2006, pp. 3227-3229.

[8] J. P. Tock et al., "The preparation of the cryomagnets and assembly of the LHC test string 2," in Proc. Particle Accelerator Conf., Chicago, 2001, pp. 3433-3435.

[9] K. Foraz, E. Barbero-Soto, H. Gaillard, C. Hauviller, and S. Weisz, "Schedule evolution during the life-time of the LHC project," in Proc. Particle Accelerator Conf., Albuquerque, 2007, to be published.

[10] A. Poncet, P. Cruikshank, V. Parma, P. Strubin, J. P. Tock, and D. Tommasini, "Assembly and quality control of the LHC cryomagnets at CERN: Motivations, means, results and lessons learnt," in Proc. Particle Accelerator Conf., Albuquerque, 2007, to be published. 